\title{
ADIPONECTIN RESPONSE TO VEGETARIAN DIET IS GENDER-DEPENDENT AND INVERSELY RELATED TO URIC ACID
}

Marijana Vučić Lovrenčić ${ }^{1}$, Iva Košuta ${ }^{1}$, Marko Gerić², Goran Gajski² and Verica Garaj-Vrhovac²

${ }^{1}$ Merkur University Hospital, Zagreb, Croatia

${ }^{2}$ Institute for Medical Research and Occupational Health, Zagreb, Croatia

\section{Objectives}

Beneficial influence of vegetarian dietary habits in reducing common risk factors of metabolic syndrome has been recently evidenced. However, adiponectin production and secretion has been scarcely studied in vegetarians, despite it's important potential in recovering metabolic homeostasis by reducing inflammation and insulin resistance.

The aim of this study was to evaluate the influence of vegetarian diet on serum adiponectin levels and it's association to the established inflammatory and metabolic biomarkers.

\section{Methods}

Total serum adiponectin (ADN), leukocytes (L), CRP, plasma glucose (PG), insulin (INS) and uric acid (UA) were measured at fasting in healthy, non-obese, age-matched vegetarian ( $N=40 ; M / F=16 / 24)$ and omnivore subjects $(N=39 ; M / F=15 / 24)$.

ADN was determined by an immunoturbidimetric method (Randox Laboratories, Ireland) and INS with a chemiluminiscent immunoassay (Advia Centaur-XP, Siemes Diagnostic Solutions, USA). HOMA-2 model was used for the assessment of betacell function (BS), insulin sensitivity (IS) and insulin resistance index (IRI).

\section{Results}

In comparison to controls, significantly lower INS $(P=0,042)$ and IRI $(P=0,041)$, as well as higher $B S(P=0,019)$ were found in vegetarians subjects.

Serum ADN leves were significantly higher in female vegetarians than the respective omnivore controls $(P=0,017)$, whereas no dietary-associated difference was observed in male vegetarian and omnivore subjects, respectively $(P=0,898)$.

Stepwise multiple regression anaysis identified uric acid as the significant negative determinant of ADN in vegetarians $\left(r_{\text {partial }}=-0,4585, P=0,002\right)$, while in omnivore subjects only $B M I$ was found to be significantly associated to ADN levels $\left(r_{\text {partial }}=-0,4439, P=0,016\right)$.

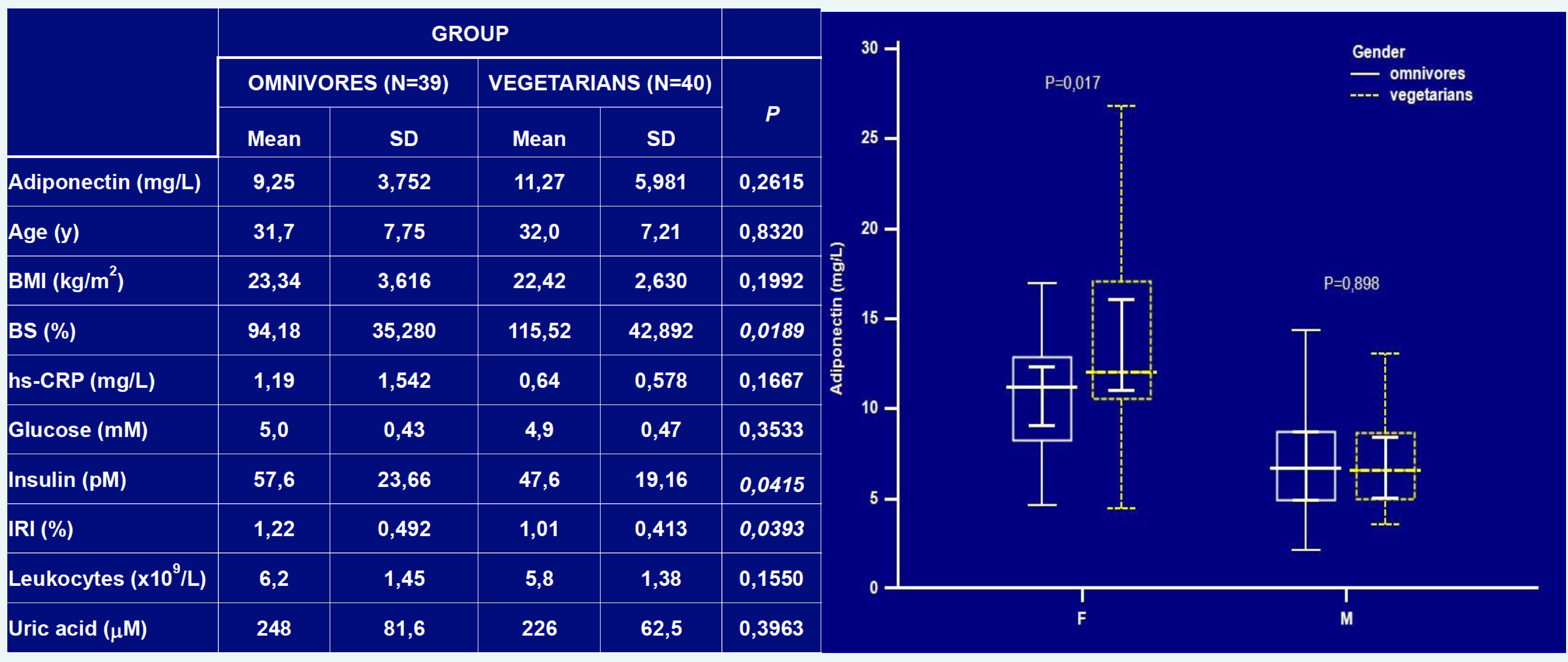

\section{Conclusions}

Vegetarian dietary habits result into improved insulin sensitivity and beta-cell function. Gender diversity in adiponectin response indicate distinct effects of vegetarian diet to adipose tissue metabolism, with a more favorable metabolic pattern in female vegetarians.

\section{References}

1. Lundsgaard A-M, Kiens B. Frontiers in Endocrinology. 2014;5:195. doi:10.3389/fendo.2014.00195. Kahleova H, Matoulek M, Malinska H, et al. Diabetic Medicine. 2011;28(5):549-559. doi:10.1111/j.1464 5491.2010.03209.

Ambroszkiewicz J, Klemarczyk W, Gajewska J, et al. Med Wieku Rozwoj. 2011 Jul-Sep;15(3):326-34

\section{Funding}

This work was supported by the Croatian Ministry of Science, Education and Sports (Grant Nos. 022-0222148-2125/045-1191348-0139).

Correspondence to: vucic@idb.hr 